Review

\title{
From Donor to Recipient: Current Questions Relating to Humoral Alloimmunization
}

\author{
Antoine Prigent ${ }^{1,2}$, Nicolas Maillard ${ }^{1,3}$, Léna Absi ${ }^{2}$, Chaker Aloui ${ }^{1}$, Fabrice Cognasse ${ }^{1,2}$, \\ Sandrine Laradi ${ }^{1,2}$, Christophe Mariat ${ }^{1,3}$ and Olivier Garraud ${ }^{1,2, *}$ \\ 1 GIMAP-EA3064, Université de Lyon, 42023 Saint-Etienne, France \\ 2 EFS Auvergne-Loire, 42023 Saint-Etienne, France \\ 3 Service de Néphrologie, Dialyse et Transplantation Rénale, Hôpital Nord, CHU de Saint-Etienne, \\ PRES Université de Lyon, 42270 Saint Priest en Jarez, France
}

* Author to whom correspondence should be addressed; E-Mail: olivier.garraud@univ-st-etienne.fr.

Received: 30 October 2013; in revised form: 16 January 2014 / Accepted: 29 January 2014 /

Published: 20 February 2014

\begin{abstract}
Alloimmunization is an undesirable iatrogenic effect of transfusion and transplantation. In fact, recipients can be considered as responders or not responders, in a continuum from tolerance, including organ transplantation and transfusion, to polyimmunized and refractory patients. New models and large studies have enabled a better understanding of the mechanisms that induce specific alloantibody (alloAb) generation. Here, we focus on risk factors of alloimmunization. We review the alloantibody characteristics, summarize the different leukocytes involved in their induction, and suggest some hypotheses.
\end{abstract}

Keywords: transfusion; transplantation; alloimmunization; tolerance; HLA

Abbreviations: Ab: antibody; Ag: antigen; APC: antigen presenting cell; $\mathrm{BC}$ : blood component; Breg: regulatory B cell; Treg: regulatory T cell; GVHD: graft versus host disease; HLA: human leukocyte Ag; HPA: human platelet Ag; MHC: major histocompatibility complex; NHFTR: non hemolytic febrile transfusion reaction; NIMA: non inherited maternal Ag; PC: platelet component; RBC: red blood cells; RBCC: red blood cell component; TRALI: transfusion related acute lung injury 


\section{Introduction}

It is estimated that every year nearly 100,000 patients benefit from organ transplantation, 20,000 from allogeneic hematopoietic stem cell transplantation, and more than 100 million from blood transfusions (http://www.who.int/en and http://www.worldmarrow.org) worldwide. Most of these medical interventions succeed and allow patients to survive their causal disease, but some recipients develop immunization against these foreign tissues, leading to rejection or impaired efficiency. Most of the alloimmunizations are Ab mediated. Alloimmunizations can occur in three ways: red blood cell component (RBCC) transfusion, in which alloimmunization rarely occurs despite the absence of an immunosuppressive regimen and frequent mismatching; organ transplantations, such as kidney grafts, in which patients can already be chronically alloimmunized against the ubiquitous foreign HLA Class I and/or the more restricted HLA Class II Ags.

Alloimmunization presents similarities and differences with infectious immunization. Both of them are influenced by $\mathrm{Ag}$ immunogenicity, physiology of high affinity Ab generation and inflammatory environment. However, alloimmunization occurs at a lesser rate than heterologous immunization and targets only few Ags, notably HLA Class I or II molecules (Table 1). There is a lack of reviews that look at all types of alloimmunization. In the first part, we here review the immunogenicity of the different alloAgs and the physiology of humoral post transfusional alloimmunization, and maternofetal immunization. In the second part, we focus upon Abs in transplantation, regulatory cells and the case of operational tolerant patients.

Table 1. Main alloimmunizations in clinical practice and comparison with heterologous immunization.

\begin{tabular}{|c|c|c|c|c|}
\hline \multirow{2}{*}{ RBC } & $\begin{array}{c}\text { Immunosuppression } \\
\text { Regimen }\end{array}$ & Immunization Rate & Main AlloAb Specificity & HLA Expression \\
& No & Moderate & RBC Ag RH1 and KEL1 & \multirow{2}{*}{ No HLA * } \\
\cline { 2 - 4 } & No & Low or very low & Other RBC Ags & HLA Class I /II \\
\cline { 2 - 4 } Platelets & Ho & Very low & HPA & HLA Class I/II \\
\hline \multirow{2}{*}{$\begin{array}{c}\text { Renal } \\
\text { transplantation }\end{array}$} & Yes & Highly frequent & \\
\hline \multirow{2}{*}{$\begin{array}{c}\text { Heterologous } \\
\text { immunization }\end{array}$} & No & Nearly $100 \%$ & Large & No \\
\hline
\end{tabular}

* note that transfusion products are contaminated by Class I/II expressing WBC.

\section{The Immunogenicity of Foreign Ags}

Three ways of immunization can lead to alloAb generation: transfusion (RBC or PC), transplantation and pregnancy. These three pathways lead to subtle differences in alloimmunization, which is poorly explored and explained. The targeted HPA specificities are different if the immunization originates from pregnancy or $\mathrm{PC}$ transfusion and $\mathrm{Ab}$ subclasses repartition varies. After a transfusion, alloAg may be captured by dendritic cells and presented to B cells (native form) and $\mathrm{T}$ cells (peptide fragments bound by HLA Class II). If inappropriate co-signaling occurs, high affinity Ab-producing plasma cells will be generated in the germinal center of secondary lymphoid organs. In 
pregnancy, the same mechanism will produce these cells, notably if fetal cells enter into the maternal circulation. Transplantation is more complex, even if indirect recognition shares some similarities with transfusion alloimmunization.

Transfusion, especially red blood cell (RBC) transfusion ( $80 \%$ of the transfusion activity), is possible because, more than a century ago, Landsteiner discovered that there were, three major groups of blood, allowing the matching of blood without creating accidents (the ABO Ags). Several decades later, another blood group was acknowledged as being strongly immunizing, the Rhesus Group. The major Ag of this group, the RH1 Ag, is assumed to be the most immunizing RBC-restricted Ag, as it easily induces immunization. Because an immunization of more than $50 \%$ is not acceptable, measures to prevent exposure of patients negative for this Ag are taken whenever possible. Interestingly, studies have shown a high variability in the rate of immunization against RH1, suggesting the involvement of other factors [1,2]. A series of observations of transfused patients revealed the potential of immunization being associated with other RBC Ags; about 20 polymorphic molecules have the capacity of immunizing a significant number of recipients (but always less than $10 \%$, which is the case for KEL1, RH3, RH4, JK1 and FY1, for others the rate is lower than $1 \%[3,4]$ ), while the remaining 300 are have not been included in the statistics. Beside RBCC transfusion, platelet component (PC) transfusion can induce immunization. PCs account for about $10 \%$ of the blood components (BCs) transfused. However, in our experience, PC transfusion is responsible for nearly half of the reported transfusion hazards. Furthermore, platelet Ags can immunize recipients, and two major types of platelet Ags must be considered. First, platelets display HLA Class I Ags, which are probably partly adsorbed from the soluble HLA molecules and partly inherited from the megakaryocytes [4,5]. Importantly, platelet HLA Class I Ags are less immunogenic than their counterparts expressed on leukocytes [6]. Second, platelets express human platelet Ags (HPA system), which are involved in achieving adhesion of platelets to the vessel epithelial wall and in hemostasis; their range of variability is much more restricted than that of the HLA system, and their immunogenicity is less frequent [7].

Multi-transfused immunocompetent recipients, such as people suffering from platelet diseases, can develop immunization in both these systems: for example, $25 \%-50 \%$ of Glanzmann's thrombasthenia patients develop anti-HLA and/or anti-HPA Abs [8,9], sometimes evolving to refractory states. It is clear that Ag immunogenicity is variable, depending on the recipient's HLA system, probably through its specific peptide presenting ability.

Some groups have identified HLA alleles association with susceptibility to immunization towards defined Ags. For example, HLA DRB1*04 and DRB1*15 are associated with immunization to the FY1 Ag [10,11], HLA-DRB1*01 and DRB1*10 are associated with immunization to the JK1 Ag [12], and DRB1*11 and DRB1*13 are associated with immunization to the KEL1 Ag [13]. Therefore, antigen immunogenicity varies depending on the receiver HLA typing, as some HLA molecules are better fitted to present an immunogenic peptide than others, while RH1 is probably more immunogenic, because it is a truncated protein, so numerous peptides can be presented by the recipient immune system.

Finally, the case of anti-HPA1a alloantibodies during pregnancy illustrates this concept, because HPA1 is a single amino-acid substitution and HLA-restricted. HPA exhibits two alleles: HPA1a and HPA $1 b$. Nearly $10 \%$ of HPA1b/b mothers have been reported to develop anti-HPA1a Abs, and $85 \%$ of anti-HPA Abs are anti-HPA1a. These Abs can induce neonatal alloimmune thrombocytopenia, which occurs in nearly one of 1,250 pregnancies [14], with a risk of intracranial hemorrhage of the 
fetus/newborn (in 7\%-26\% of the cases). The mother's HLA typing seems to have a decisive influence on the immunization risk to HPAla: ninety percent anti-HPAla immunized mothers carry HLA-DRB3*01:01, while only $30 \%$ of the mothers carrying both HPA1b/b (thus can develop anti-HPA1a) and HLA-DRB3*01:01 develop anti-HPA1a Abs indicating that other factors are involved. Thus, HLA-DRB3*01:01 seems to be necessary but not sufficient for inducing anti-HPA1a immunization. Recipient HLA restriction of donor HLA Class I molecules is hardly difficult, as it is highly polymorphic (both HLA and peptide fragment).

In connection with these anti-HLA Abs, some laboratories have started to consider epitopes rather than antigens, and even eplets rather than epitopes. Eplets are key polymorphic components of the donor HLA epitope. It has been demonstrated that some anti-HLA Abs bind one or two eplets of the donor HLA. One at least contains a HLA mismatch between the donor and the recipient [15].

In summary, immunogenicity depends on differences between donor and recipient (single amino acid polymorphism is less immunogenic than truncated protein), and on restriction by the HLA system of these differences (considering the fit for the mismatched peptides).

\section{Alloantibodies in Transfusion}

Alloimmune-induced responses to blood cell Ags are chiefly Ab-based, and more precisely IgG in nature, indicating that they result from conventional T-dependent B-cell activation and differentiation. $\mathrm{Ab}$ responses to alloimmunization against peptide blood cell Ags also differ from the so called "natural" $\mathrm{Ab}$ responses to carbohydrate moieties referred to as $\mathrm{A}, \mathrm{B}$ (ABO system) and some other Ags, which originate from $\mathrm{T}$-independent immune responses involving cross-reactive stimulation of extra follicular B cells by bacteria from the intestine. Low affinity IgM Abs are thus produced from iso- and not alloimmunization (these IgM Abs, however, mediate serious erythrocyte lesions responsible for massive hemolysis and cytokine storm). Natural anti-HLA Abs, probably induced by cross-reactions, are detected in some cases: some subjects share "natural" Abs against some HLA alleles without previous reported exposition. Such Abs have been poorly studied, and lab testing has demonstrated highly heterogeneous rates between patients (recipients), probably because of technical lab issues (e.g., cutoff values and methods used for detection) [16]. Some laboratories defined cutoff values on the basis of anti-HLA Abs found in healthy blood donors. The origin, significance, and clinical relevance of "natural" Abs have been poorly studied. Cross-reactions with environmental Ags are probable, and Ag denaturation in detection methods can be involved, as it exhibits some cryptic epitopes.

In the aggregated state, the model derived from most infectious agents would predict a strong stimulation and a large-scale immunization. For transfusions this is not the case, as the most immunizing epitope in transfusions is derived from the RH1 protein, which elicits a detectable immune responses in nearly $50 \%$ of cases. Unless transfusion is chronically repeated, for example in sickle cell disease patients, the frequency of detectable humoral immunization is nearly $25 \%$, a rate much smaller than the theory would predict.

Besides the apparently stringent HLA restriction, co-signaling is known to dampen Ag presentation and APC differentiation. Because Signal 1 is probably effective, absence of inflammation - in blood products or in the recipient state-would possibly impair the alloimmune response. This raises the possibility that limited inflammation is responsible for reduced immunization, and that general genetic 
predisposition modulates the susceptibility to inflammatory signals. Indeed, Signal 1 without Signal 2 leads to anergy or clonal deletion. Concerning Ab responses in terms of classes and subclasses, Ags from infectious pathogens are commonly tested for, while this is not the case for blood cell Ags.

\section{Donor and Blood Product Related Factors in Transfusion}

\subsection{Inflammation and Alloimmunization}

Additional, parallel findings indicate that in experimental models, immunogenicity of Ags can be enhanced by creating an inflammatory environment [17]. Immunogenicity is intrinsically linked to inflammation. Inflammatory factors in blood products can occur in two ways: "preparation", which might influence proinflammatory molecule freeing during storage (i.e., when there is no leukoreduction), and potentially "dangerous donors", which might express a high rate of proinflammatory factors that stay or accumulate in blood products. Blood products indicate that inflammation is essential for immunization. In real transfusion conditions, inflammation is generally sterile, and the inflammatory response is created by several endogenous stimuli, including altered or ageing cells (RBCs, platelets), leucocytes and their residues, microparticles from different cell types, cytokines, chemokines and generally all molecules that are now ascribed as biological response modifiers (BRMs). The question of the origin of these BRMs is not clear, and there are probably multiple origins: if leucocytes are the most important providers of BRMs, contribution of each type has to be better characterized; in fact, platelets are involved in the production of BRMs, in soluble form and/or into microparticles. Finally, RBCs or long-living soluble plasmatic molecules might contribute to some detrimental effects.

It must be noted that BRMs can be infused with plasma or secreted in copious amounts by stored platelets [18]. In addition, stressed erythrocytes have been shown to present themselves with danger signals that elicit the production of proinflammatory BRMs by circulating or vessel-lining leukocytes, and by vascular endothelial cells [19]. Furthermore, it has been shown that platelets and their secreted cytokines can alter the capacity of B lymphocytes to differentiate and bias the non-specific (polyclonal) production of Ig in terms of isotypes, at least in experimental models [20]; they may also influence Ag presenting cells (such as DCs) of the recipient. The role of iron in creating inflammatory conditions in stored RBCCs is still disputed. Iron-mediated inflammatory conditions have been proven [21], and iron is released during storage, but aged (versus fresh) RBCCs do not seem to enhance alloimmunization in large-scale studies [22]. Recently it has been suggested that the collection and preparation mode of RBCCs could affect the proinflammatory status of the RBCC or the single donor apheresis platelet component in ex vivo experiments. However, clinical data do not support this finding. If platelets, plasmatic molecules or RBC can generate some BRMs, the main contributors are probably residual leukocytes, notably if no leukoreduction has been performed. In this way it is interesting to note that leukocytes and platelets seem to act in a synergistic manner in anti-HLA immunization [23]. Even with a leukoreduction, this immunization occurs essentially against Class I (expressed by platelets and leukocytes) and Class II (not expressed by platelets) HLA. Inversely, this synergy is not observed in RBC transfusion [24]. 


\subsection{Residual Leukocytes and Alloimmunization}

A blood product transfused to a recipient is composed of three distinct parts: (1) the desired product(s), such as RBCs or platelets; (2) some excipients - comprising anticoagulant, residual plasma and additive solutions; (3) residual leukocytes and leukocyte debris that carry HLA and non-HLA Ags, and occasionally unexpected residual cells, e.g., RBCs in PCs, and platelets in RBCCs. Some data link the presence of residual allogeneic leukocytes (potentially inflammatory factors secreting cells) with alloimmunization against RBC or platelet Ags [25,26]. Intensive leukoreduction has been experimentally shown to favor tolerance (or at least absence of $\mathrm{Ab}$ formation) instead of alloimmunization [25,27]. Several groups have shown that highly purified blood cell preparations infused in mice are weakly immunogenic when leukocytes are totally absent from the suspension [28]. Interestingly, there seems to be a differential role for MHC Class II negative and positive cells (such as B lymphocytes): in one study, B lymphocytes helped minimize alloimmunization to MHC Class I Ags in mice [29], while these data have been challenged by others [30]. Discrepancies between data may stem from protocol variations and viability (and/or experimental alteration) of leukocytes, eliciting either direct or indirect Ag presentation [31].

In regard to platelets, the trial to reduce alloimmunization of platelets (TRAP) (1997) provided insightful data on HLA Class I platelet immunogenicity itself, as it was clearly shown that leukoreduction (and/or leukocyte inactivation by UV irradiation) significantly reduced alloimmunization, though it did not abrogate it [25]. The HLA Class I immunization potential of platelets is large (though less frequent than leukocytes, about $18 \%$, compared to more than $45 \%$, respectively [25]). On the other hand, we may consider that more than $80 \%$ of PC are non-immunizing in patients, despite the fact that in the vast majority of cases there is Ag discrepancy (mismatch) between donor and recipient regarding their respective HLA Class I phenotypes. Importantly, HLA Class I in platelets often lack $\beta 2$-microglobulin, and this incomplete HLA could be adsorbed from plasma soluble forms. During storage, platelets' HLA is released from the membrane in soluble forms, enhancing complexity, as soluble molecule freeing is suspected to be linked to the transfusion related immune modulation (TRIM) effect of platelet products, although soluble forms alone cannot induce it $[32,33]$. Leukocytes also mediate TRIM to dampen inflammatory responses and alloimmunization, inducing tolerance mechanisms such as clonal deletion of reactive T-cells, elicitation of regulatory T-cells, anti-idiotypic $\mathrm{T}$ and B-cell responses, suppression of Natural Killer cell activity, switching from Th1 to Th2 type responses, selection of non-responder type immune cells, induction of apoptosis and favoring accumulation of regulatory factors, such as soluble MHC Class I molecules [34,35]. Allogeneic transfusion transfers residual donor leukocytes including DCs with the ability to present Ags. In the case of the DCs, they share at least one HLA (Class II) molecule with the recipient's immune cells, and tolerance is induced. In contrast, fully HLA mismatched transfusions are more likely to induce alloimmunization [36]. Thus, leukocytes exert a dual function in transfused patients, and it is difficult to state whether leukoreduction is beneficial or eventually detrimental for alloimmunization induction, which is currently debated. However, leukoreduction seems beneficial for many other reasons, such as reduction of discomfort, inflammatory acute transfusion reactions, NHFTR, TRALI cases, GVHD cases and transfusion transmitted infections. 


\section{Recipient Related Factors in Transfusion}

There is evidence that an inflammatory state in the recipient favors the occurrence of alloimmunization in mice [37-39] and possibly in humans [40], and that some recipients are responders (they respond easily to alloimmune stimulation) while others are non-responders.

\subsection{Genetic Risk Factors}

We have discussed the role of HLA, but HLA restriction is limited to certain alloAgs, which apparently are best fitted to take place in the HLA groove. In recipients, other factors can affect the alloimmune response. Although poorly explored (TRIM 21 and CD81 have been proposed [41,42]), non-HLA genetic factors could be involved, though not necessarily in an amino acid modification manner. CD81 has been shown to be involved in the alloimmune response against RBC Ags in sickle cell disease patients, suggesting that the immunogenicity is linked to an inherited predisposition to trigger a humoral immune response through intronic polymorphisms, modifying the expression of key costimulatory proteins.

\subsection{Environmental Risk Factors}

Environmental factors, such as some inflammatory states, can also influence alloimmunity. Several well-documented studies have clearly shown that experimental immunization does not occur in the absence of inflammation, and, on the contrary, have indicated that this situation favors Ag-specific tolerance, sometimes in an identified active regulatory and sometimes in an immunologically ignorant way $[17,37,38,43]$. It is now well accepted that inflammation is instrumental to immunization after transfusion, and, moreover, that certain transfusion conditions induce inflammation. BCs can induce inflammation, but inflammation can precede transfusion.

\subsection{Recipients' Tolerance to AlloAgs}

Some patients do not develop alloAbs even after repeated RBC transfusions in multi-transfused patient populations, despite high frequencies of $\mathrm{Ag}$ mismatch [44,45]. In mouse models of RBC immunization [46,47], CD25+ T cells have been reported to mediate active tolerance to transfusion. Exploration of the Treg and Breg compartments in sickle cell disease patients revealed that alloimmunized patients displayed low rates of Treg cells, reduced levels of IL-10 and enhanced production of IFN $\gamma$ compared with more tolerant patients $[48,49]$. In an immune thrombocytopenia model, the absence of Treg cells favored alloimmunization against platelet donor Ags and thrombocytopenia in transfused recipients [50]. It seems that exposition to alloAgs induces both immunization with $\mathrm{T}$ helpers and Tregs, leading to alloimmunization or specific tolerance induction, respectively. This hypothesis is supported by non-HLA genetic risk factors and could be associated with apoptosis and anergic ways of tolerance. Ignorant way of tolerance appear to be unlikely in the case of RH1 Ag, as a large amount of Ag is transfused and numerous potential presented peptides are mismatched. Concerning other mismatches with single amino acid substitution, ignorance is possible and supported by HLA restriction. Overall, alloimmunization can be viewed as a breach of tolerance. Consistently with these remarks, autoimmunity and alloimmunity against RBC have been linked 
together in some studies [44,51]. Concerning platelet transfusion, alloimmunity and autoimmunity seem to also be associated in some cases [52].

In summary, multiple immunizations render transfusion programs extremely difficult to achieve and compromise survival of exposed patients. We propose a hypothesis to explain these progressive immunizations, which are not exclusive: first, multiple $\mathrm{BC}$ transfusions will heighten exposure of the recipient's immune system to "unknown Ags"; second, a large amount of delivered Ag can induce newly moderately fitted HLA-peptide combinations on recipient presenting cells; third, environmental conditions, i.e., inflammation, can breach previous tolerance by delivering inappropriate co-stimulation signals; finally, recipients can have a high susceptibility to alloimmune responses.

\section{The Pregnancy Example}

The placental immune privilege is an efficient mechanism of tolerance, preventing alloimmunization against paternal Ags inherited by the newborn. In fact, high affinity IgG generation and $\mathrm{T}$ cell mediated cytotoxicity need a classical Ag presentation by HLA Class I and Class II, which is not allowed by trophoblastic cells, which express HLA-G to avoid natural killer cell cytotoxicity. HLA-G is a non-classical and non-polymorphic HLA molecule, so it cannot present mismatching. Unwanted obstetrical events can nevertheless induce a breach in this barrier, exposing the mother to RBCs, WBCs or platelets from the fetus in her own circulation, initiating T-dependent B cell responses and active maternal IgG based immunization against inherited paternal Ags. In pregnancy, HLA, RBC Ags and HPA can induce alloAb formation. Although anti-HLA Abs are frequently detected, they are not (or ony few) pathological, while anti-RBC Ag and anti-HPA Abs are rare, but can provoke severe fetal disorders.

Immunization against HLA increases with pregnancies, $12.1 \%-48.5 \%$ (depending on methods, cutoff values and interval of last pregnancy) of females having carried three children or more exhibit detectable Abs (Class I and/or II) [53] without clinical detrimental consequence. In contrast, only 1/1,000 pregnant women exhibits anti-HPA Abs, mostly pathological [14,54,55]. Fetus/newborn hemolytic disease, often due to pathological maternal anti-RH1 (in RH1 negative populations), is decreasing since treatments with therapeutic Abs have been initiated. These Abs are also anti-RH1 Abs, but as they reduce the fetal RBCs lifespan, they avoid maternal immunization. Mothers can be also actively immunized by fetal platelets. Contrary to immunization against main RBC Ags, they may develop immunization anti-HPA during the first pregnancy, because HPA Ags are expressed as soon as week 16.

An additional factor besides the RH1 or HPA mismatch and HLA restriction may be the ABO phenotype (platelets display few copies of A and B Ags), which seems to affect the outcome and the severity of thrombocytopenia of the newborn [56] while it may reduce RH1 Ag immunization by reducing the fetal RBCs lifespan [57]. Isotypes of anti-HPA or anti-RH1 specific Abs have not been clearly linked with pathogenicity [58]. Finally, we have to consider fetal tolerance against maternal Ags, which has been highlighted by NIMAs tolerance. In fact, the fetal immune system more easily tolerates the mother's HLA, even if it is not transmitted. This point will be discussed below. 


\section{Antibodies in Transplantation}

Renal transplantation is mainly discussed in this section, because the kidney is the most frequently transplanted organ and its rejection is of important clinical interest.

\subsection{Donor-Specific Abs and Rejection}

Candidates for transplantation often produce Abs against HLA Ags. "Natural" Abs are infrequent and Abs mostly result from alloimmunization following multiple exposures to leukocytes or platelets (blood transfusion, pregnancy), or to previous organ transplants [59]. Pre-existing alloAbs can cause hyperacute organ rejection immediately after transplantation, leading to the destruction of the graft within a few days, if targeting donor-specific Ags (DSA). De novo produced DSAs (post transplantation) may appear later in the recipient (mean time from 8 months to 4 years after transplantation, but can appear in the first weeks after transplantation [60]), in this case leading to acute or chronic Ab-mediated rejection (AMR), which in general, progressively impairs grafted organ functions [61]. Considerable achievement in diagnosis has recently emerged, as the single beads technology enables the detection of low rate and early DSAs. Indeed, DSAs are frequently associated with the so-called C4d-positive rejection in renal biopsies (related to humoral-mediated rejection, as $\mathrm{C} 4 \mathrm{~d}$ is a fragment of the complement system), while C4d-positive rejection is rarely DSA negative [62]. However, for reasons that are not yet fully understood, not all DSAs cause graft dysfunction [63,64], though some of them lead to subclinical rejection. Finally, a fraction of rejections are mediated by cellular cytotoxicity. The mechanisms of donor-specific Ab toxicity mostly involve two ways. The first is complement activation by the classical pathway, which triggers inflammation, recruitment and cell damage, and subsequently $\mathrm{C} 4 \mathrm{~d}$ deposition in renal graft microcirculation. The second is the Ab-dependent cell-mediated cytotoxicity of NK cells, which stimulates their cytotoxicity abilities (via killer activation receptors (KAR)), especially because of the lack of killer inhibitory receptor (KIR) ligands on transplanted cells. The $\mathrm{Ab}$ titration probably has prognostic significance, but needs more standardization.

Isotypes (Ig classes) and subclasses of DSA have been explored, relative to DSA cytotoxicity and graft outcomes. It is now established that IgGs are probably the only deleterious isotype. In some cases, IgMs are detected, but they are poorly related to graft outcome [62]. IgAs are frequently detected, but a correlation with graft survival has to be established [65]. Neither IgE nor IgD have been reported. Overall, it is generally considered that pathogenicity is resumed by IgGs. DSA IgGs of different subclasses have been evaluated, without clear association of any subclass with graft survival. IgG1 and IgG3 (the two complement activating subclasses) were expected to be more involved in rejection than $\mathrm{IgG} 2$ and $\mathrm{IgG} 4$, however these non-complement activating subclasses are not associated with better prognosis, suggesting other mechanisms of rejection [61,66]. The causality of immunization (pregnancy, BCs transfusion or graft) seems to have a low impact on the subclass repartition [66], and subsequently on the pathogenicity. In general, subclass analysis is not considered clinically relevant in this matter.

Years ago, the fine analysis of specificity of anti-HLA DSAs was problematic, because target cells generally harbored multiple HLA Ags. This difficulty in testing made the detection of multiple 
immunizations harder. Moreover, poly-immunized patients could be tested positively for all target cells used in the labs. The emergence of single bead technology enabled better characterization of DSA specificity, because one bead stands for one allele (apart for the polymorphic DQA, which forms a heterodimer with the polymorphic DQB1). Abs against Class I HLA-A and -B, and Class II HLA-DR and HLA-DQ are clearly associated with AMR. However, the association between graft survival and DSAs against HLA-C, HLA-DQA, HLA-DRB3/4/5 and HLA-DPB1 is less clear [67-71]. The first explanation is that these Ags were difficult to explore before single bead technology and consequently less documented. The second explanation is that certain Ags are weakly expressed and therefore elicit poor immune responses and the corresponding Abs are rarely present, with low rates (when detectable), and associated with other anti-HLA Abs. Hence, the clinical significance remains elusive.

\subsection{Non-HLA Abs}

The hypothesis of a non-HLA component in rejection was developed during the time of indirect recognition (except natural $\mathrm{ABO} \mathrm{Abs}$ ) and seems to be consistent with the idea of an intrinsic predisposition to rejection. Some recipients' polymorphisms related to the innate immune system (e.g., cytokines, toll-like receptors (TLRs) and damage-associated molecular patterns (DAMPs) are interesting candidates for explaining the inherited predisposition favoring the alloimmune response, regardless of specificity, however it is still heavily debated [72]. Moreover, previously immunized patients, grafted with HLA identical donors, show greater rates of chronic rejection [73]. However, an acquired sensitization against non-HLA polymorphisms is another possibility.

In the past few years, a regain of attention focused on non-HLA alloAbs in organ transplantation as potential targets, especially on anti-endothelial cell Abs (endothelium being the main interface between the recipient's immune system and the grafted tissue). HLA Class I and II molecules are not the only proteins with high polymorphisms in humans. A potential target is the non-HLA but MHC-related protein, MICA (major histocompatibility complex class I-related chain A), which is encoded by the $M H C$ gene. Indeed, $M H C$ genes comprise HLA and other proteins, like MICA, which spans a highly polymorphic protein family included in the Class I MHC genes, as Class I HLA. MICA can be targeted by specific alloAbs, with consequent correlation with renal graft outcome [74], however a routine exploration is still discussed. Interestingly, Ags other than MICA and HLA have been proposed [75]. In summary, the clinical relevance of Abs against non-HLA Ags has to be further demonstrated in large cohort prospective studies [62].

Auto-Abs are frequently detected after transplantation, and are more frequent in rejector than non-rejector grafted patients. One reason would be that some rejectors are prone or predisposed to elicit deleterious humoral responses. Another factor would be some influence of alloimmunity on autoimmunity in such patients, like epitope spreading or cross-reactions. Some studies have tried to determine Abs with clinical value, but Ags targeted by AMR-related auto-Abs vary and are rarely reported in the different studies [76]. Thus, the involvement of auto-Abs in graft overcome is not well established, as so far only one of them has been shown to have a pathologic role, the anti-angiotensin II receptor type I Ab, which may lead to hypertension [77].

Considering bone marrow transplantation, autoantibodies are frequently detected, due to the cooperation between donor's and recipient's lymphocytes, leading to somatic hypermutation and class 
switching. Thus, recipient CD8 T cells kill donor lymphocytes, and donor CD8 T cells kill recipient lymphocytes, enhancing complexity. The relations between autoimmunity and alloimmunity often occur through HLA and TCR, in the two ways.

Finally, ABO Abs have to be mentioned for two reasons: ABO Abs are a barrier for renal transplantation, although increasingly discussed now [78]. However, donors' passenger leukocytes can induce Abs against the recipient ABO system, in some cases leading to hemolytic anemia [44,79].

\section{Regulatory Immune Cells in Transplantation}

Inflammatory cells such as cytotoxic T cells, helper T cells, mature dendritic cells and M1-type macrophages enhance immune responses and cooperate to induce cellular mediated cytotoxicity and humoral anti-donor Ag-specific responses. This "inflammatory network" (not reviewed here) has a regulatory counterpart, which does not seem to be sufficient to overcome inflammation in current organ transplantation. Thus, an immunosuppressive drug regimen is still necessary to avoid organ rejection. Nevertheless, regulatory leukocyte populations in transplantation have been extensively studied and described for 20 years. Two leukocyte types have been proven to be particularly involved in tolerance: the first are leukocytes that have acquired regulatory properties during development in their local microenvironment, such as natural Treg (nTreg) cells [80]; the second are naïve leukocytes that differentiate peripherally into regulatory cells, such as induced Treg (iTreg) cells [81,82]. The "regulatory network" gains complexity over time and affects both cellular and humoral immunity. New leukocyte populations/subpopulations join in progressively, in innate as well as in adaptive immunity [83].

\subsection{Adaptive Immunity}

Adaptive immunity relative to organ transplantation reveals great complexity: induced and natural CD4+ Foxp3+ regulatory $\mathrm{T}$ cells are the main regulatory populations [84,85], while less frequent regulatory $\mathrm{T}$ cell populations such as $\mathrm{CD} 8+$ regulatory $\mathrm{T}$ cells $[84,85], \operatorname{Tr} 1 \mathrm{~T}$ cells [86], double negative $\mathrm{T}$ cells [87], NKT cells [88] and $\gamma \delta \mathrm{T}$ cells $[89,90]$ are also involved in transplantation tolerance [83]. Some of these rare populations can be induced by iTreg cells (CD8+ regulatory $\mathrm{T}$ cells and $\operatorname{Tr} 1$ ), while others appear independently (nTreg, double-negative Treg). These regulatory cells are involved in tolerance and have been detected in recipients, but are insufficient for preventing organ rejection, especially when memory $\mathrm{T}$ cells against donor antigens are present [91]. Interestingly, iTreg cells are induced by the graft and remain at detectable levels after rejection [92].

Besides regulatory $\mathrm{T}$ cell populations, there has been a more recent report on regulatory $\mathrm{B}$ cells (Breg). Breg populations have been detected in operational tolerant (OT) patients, as IL-10 or TGF $\beta$ secreting B cells with transitional T1/T2 immunophenotype (CD24+CD38+) [93-95], and have been proposed as iTreg cell inducers. B cell-induced iTreg cells seem to have powerful suppressive activities in transplantation [96]. The specificity of Breg cells has not been extensively explored yet, as well as their Ab producing capacity, class switching, affinity maturation, or plasma cell differentiation. It is thought that mainly Breg cells are B10, a heterogeneous B cell population that secrete IL-10 in large amounts to induce their regulatory activities in a CD40/BCR dependent manner. Furthermore, alloimmune tolerance can be induced by other B cell types, which could be IL-10 non-secreting cells, 
such as transitional B cells. Another one could be IgM+ B cell clusters that have been reported in "regulatory clusters" of tolerant rats [97].

In humans, B cell recognition consistently occurs early after the graft, despite immunosuppression, without triggering an alloimmune response and rejection [98], but the regulatory activities of these clusters needs more investigation. Finally, the CD40 pathway has been proven necessary to activate the alloimmune adaptive system, but its absence protects from rejection [99,100]. CD40 is present on B cells, and its ligand, CD154, is present on T cells (both not exclusive). The CD40/CD154 pathway is considered fundamental in $\mathrm{Ag}$ presentation co-signaling and tolerance, it is also an essential pathway for activation and differentiation of Breg. Anti-CD40 and anti-CD154 monoclonal Abs are currently tested as kidney rejection treatments.

\subsection{Innate Immune System}

As for adaptive immune cells, the heterogeneity and plasticity of innate immune cells (mostly macrophages and DCs) guide them to inflammatory or regulatory contingents. The historical principle of tolerogenic immature dendritic cell versus inflammatory mature dendritic cell populations is insufficient to resume innate immunity in alloimmune intervention. A similar sub-specialization has been described for macrophages.

Macrophages were detected a long time ago as one of the dominant infiltrating cells in rejected grafts [101]. They are related to poor graft outcome [102,103] and atherosclerosis [104]. Macrophages are plastic cells with a large range of functions that depend on their activation state from M1 or M2 [105,106]. Recently, a third type of macrophage, regulatory macrophages (Mreg), has been suggested to be split off from M2 macrophages [106]. M1 macrophages are involved in phagocytosis and inflammation, while M2 macrophages seem to be more related to tissue repair (desirable) and potential fibrosis (detrimental because it leads to arteriosclerosis); in this scheme, Mreg cells would control the immune suppression [83,107]. However, few studies about Mreg implications in organ transplantation have been reported $[108,109]$.

DCs are crucial cells for priming TD adaptive immunity, including tolerance and rejection in transplantation. Allospecific rejection can occur in three ways, involving DCs in each case: (i) direct recognition, where donor DCs are recognized by recipient $\mathrm{T}$ cells (donor MHC and donor peptides), mostly in acute rejections; (ii) indirect recognition, where donor peptides (notably from donor MHC) are presented by recipient $\mathrm{MHC}$ on DCs, mostly in chronic and humoral rejections; (iii) semi-direct recognition, where intact donor MHC-peptides are transferred to recipient DC membranes [110-114]. All three ways can lead to TD adaptive alloimmunity or tolerance, depending on DC state of maturation: immature DCs promote tolerance, while mature DCs activate effector cells. Conventional immature DC populations seem to induce iTreg cells, and inhibit B cells, T cells and NK cells, while mature DCs enhance specific Ab production. Two other subtypes have been explored more recently, with potential immunosuppressive activity: the myeloid derived suppressor cells and the mesenchymal stromal cells. Plasmacytoid dendritic cells can also promote tolerance and induce iTreg differentiation $[83,115]$.

In summary, numerous cellular actors have been reported to take part in tolerance, but in clinical

practice, this important network seems insufficient for preventing rejection when there is no 
immunosuppression. Further research is needed to understand the cellular cooperation and induction, as well as tolerance initiation and enhancement.

\section{Operational Tolerance (OT) in Transplantation}

Operational tolerance (OT) patients are able to tolerate a transplant despite a break in immunosuppressive therapy [116]. These are rare in kidney transplants, but are more frequent in liver grafts. Cohorts of renal grafted OT are now in progress to elucidate the tolerance mechanisms. However, the few cohorts reported so far are difficult to compare and the numbers of HLA mismatches appear heterogeneous. In addition, OT patients evolve differently after a few years: they can accommodate or support durable tolerance, or sometimes switch to chronic rejection [117,118]. Infrequently, DSAs are detectable, sometimes with high titers, but generally without activating the complement system (in biopsy staining) [116]. Interestingly, OT patients seem to be low responders to blood transfusions, reinforcing the idea of genetic predisposition to alloimmunization [118]. The physiological mechanism of tolerance in transplantation may involve regulatory $\mathrm{T}$ cells, $\mathrm{pDCs}$ and $\mathrm{mDCs}$ ratio, $\mathrm{LT} \gamma \delta$, as well as some cytokine polymorphisms, especially in TGF $\beta$ and IL-10 [119,120]. However, no sensitive and specific marker has been clearly identified, and it is difficult to discriminate between causal factors and consequences. Some studies have examined leukocyte populations and mRNA in PBMCs from dozens of OT patients, and concluded that there are B cell signatures in OT patients, and possible Breg cell involvement, through IL-10 or TGF $\beta$ secretion [94,95]. Nevertheless, the absence of immunosuppressive therapy is biased by immunosuppression in the control transplant patients, and these studies show a similar pattern between OT and healthy non-grafted patients. However, control patients given an immunosuppressive treatment presented a strong alteration of the B cell compartments, particularly at the time of rejection.

Overall, it is now clear that this is not immune ignorance or non-recognition of alloimmune Ags, but an active immunoregulatory phenomenon that permits graft tolerance. Indeed, cryptic allospecific reactions are detected in stable grafts in early days without acute rejection [98], suggesting that all patients develop a specific regulatory network, though insufficient for avoiding rejection.

Non-inherited maternal Ags (NIMAs) are interesting candidates for transplantation. In fact, the fetus tolerates the maternal HLA Ags, even if they are not inherited, because this exposition occurs in a tolerogenic context (during pregnancy). However, some authors explored graft survival comparing the father's versus the mother's kidneys, and no significant differences were found. It seems that the mother does not tolerate the inherited paternal Ags, and that some passenger leukocytes located within the graft can induce some proinflammatory signals, which would counterbalance the benefit of the NIMAs [121]. Nevertheless, the NIMAs are an interesting way to raise the tolerance in the case of NIMAs from other, previously not exposed donors are available. More studies are needed to confirm these hypotheses and to prove the benefit in large studies.

\section{PERSPECTIVES: Coming Soon the "Regulatory Plasma Cells" (Preg)?}

Plasma cells are known to be essential Ab-secreting cells of the adaptive immune response after infection. As Breg cells are a very recently characterized population, their differentiation potential has not been explored, but it can be suggested that they may differentiate and that their differentiation state 
is immunosuppressive. Why are Breg cells only considered as "immunosuppressive Ag-presenting cells", while they express a B cell receptor? Humoral tolerance may establish an evolving pathway, similarly to its immunoreactive counterpart and the ancestrally linked $\mathrm{T}$ cells.

Abs in transplantation are not always considered as pathogenic, and sometimes they are suspected as protective, including DSAs [122]. Auto-Abs binding DAMPs are evocated in tolerance [123-125]. Moreover, B cells are involved in transplants and transfusion tolerance. B cells differentiate into plasma cells or memory cells. Are Breg cells able to differentiate into immunosuppressive Ab-secreting cells?

Isotype characterization cannot discriminate between tolerance and rejection, thus other candidates have to be hypothesized. Some Ab glycoforms show increased immunosuppressive Ab capacity in conjunction with the affinity of the Fc fragments with the different FcRs, notably in autoimmunity and allergy. They may explain the efficiency of intravenous immunogobulin (IVIg) therapy, when used in treatment of autoimmune diseases and graft rejection. These glycoforms depend on the enzymatic composition of the Ab-secreting cells (i.e., alpha2,6-sialyltransferase), and the main mechanism may depend on the inhibitory FcRs (and alternate receptors) affinity [126-129].

In transplantation, some gene polymorphisms related to B cells, Breg cells and Abs are suspected in OT patients $[94,95,130]$. It can be hypothesized that a Preg cell compartment exists and takes part in the overall regulatory network, expresses alpha2,6-sialyltransferase (and/or other similar enzymatic activity), and would favor the low rate production of inhibitory glycoforms of DSAs.

\section{Concluding Remarks}

Alloimmunity is a long-term concern, as bone marrow transplantation, organ transplantation, and transfusions have no alternative. New immunosuppressive treatments have made it possible to reduce inefficiency and rejection, but they still have important adverse effects. Risk factors, biological markers and donor-recipient compatibility, as well as immunosuppressive optimization are serious candidates to reduce graft failures and adverse effects.

\section{Conflicts of Interest}

The authors declare no conflict of interest.

\section{References}

1. Urbaniak, S.J. Alloimmunity to RhD in humans. Transfus. Clin. Biol. 2006, 13, 19-22.

2. Yazer, M.H.; Triulzi, D.J. Detection of anti-D in D- recipients transfused with D+ red blood cells. Transfusion 2007, 47, 2197-2201.

3. Schonewille, H.; Brand, A. Alloimmunization to red blood cell antigens after universal leucodepletion. A regional multicentre retrospective study. Br. J. Haematol. 2005, 129, 151-156.

4. Kao, K.J.; Cook, D.J.; Scornik, J.C. Quantitative analysis of platelet surface HLA by W6/32 anti-HLA monoclonal antibody. Blood 1986, 68, 627-632.

5. Lalezari, P.; Driscoll, A.M. Ability of thrombocytes to acquire HLA specificity from plasma. Blood 1982, 59, 167-170. 
6. Gouttefangeas, C.; Diehl, M.; Keilholz, W.; Hornlein, R.F.; Stevanovic, S.; Rammensee, H.G. Thrombocyte HLA molecules retain nonrenewable endogenous peptides of megakaryocyte lineage and do not stimulate direct allocytotoxicity in vitro. Blood 2000, 95, 3168-3175.

7. Kiefel, V.; Konig, C.; Kroll, H.; Santoso, S. Platelet alloantibodies in transfused patients. Transfusion 2001, 41, 766-770.

8. Santoro, C.; Rago, A.; Biondo, F.; Conti, L.; Pulcinelli, F.; Laurenti, L.; Perrone, M.P.; Baldacci, E.; Leporace, A.; Mazzucconi, M.G. Prevalence of allo-immunization anti-HLA and anti-integrin alphaIIbbeta3 in Glanzmann Thromboasthenia patients. Haemophilia 2010, 16, 805-812.

9. Poon, M.C.; D’Oiron, R.; Von Depka, M.; Khair, K.; Negrier, C.; Karafoulidou, A.; HuthKuehne, A.; Morfini, M. Prophylactic and therapeutic recombinant factor VIIa administration to patients with Glanzmann's thrombasthenia: Results of an international survey. J. Thromb. Haemost. 2004, 2, 1096-1103.

10. Picard, C.; Frassati, C.; Basire, A.; Buhler, S.; Galicher, V.; Ferrera, V.; Reviron, D.; Zappitelli, J.P.; Bailly, P.; Chiaroni, J. Positive association of DRB1 04 and DRB1 15 alleles with Fya immunization in a Southern European population. Transfusion 2009, 49, 2412-2417.

11. Noizat-Pirenne, F.; Tournamille, C.; Bierling, P.; Roudot-Thoraval, F.; Le Pennec, P.Y.; Rouger, P.; Ansart-Pirenne, H. Relative immunogenicity of Fya and $\mathrm{K}$ antigens in a Caucasian population, based on HLA class II restriction analysis. Transfusion 2006, 46, 1328-1333.

12. Reviron, D.; Dettori, I.; Ferrera, V.; Legrand, D.; Touinssi, M.; Mercier, P.; de Micco, P.; Chiaroni, J. HLA-DRB1 alleles and Jk(a) immunization. Transfusion 2005, 45, 956-959.

13. Chiaroni, J.; Dettori, I.; Ferrera, V.; Legrand, D.; Touinssi, M.; Mercier, P.; de Micco, P.; Reviron, D. HLA-DRB1 polymorphism is associated with Kell immunisation. Br. J. Haematol. 2006, 132, 374-378.

14. Kaplan, C. Foetal and neonatal alloimmune thrombocytopaenia. Orphanet J. Rare Dis. 2006, 1, 1-6.

15. Duquesnoy, R.J. The antibody response to an HLA mismatch: A model for nonself-self discrimination in relation to HLA epitope immunogenicity. Int. J. Immunogenet. 2012, 39, 1-9.

16. Morales-Buenrostro, L.E.; Terasaki, P.I.; Marino-Vazquez, L.A.; Lee, J.H.; El-Awar, N.; Alberu, J. "Natural" human leukocyte antigen antibodies found in nonalloimmunized healthy males. Transplantation 2008, 86, 1111-1115.

17. Hendrickson, J.E.; Desmarets, M.; Deshpande, S.S.; Chadwick, T.E.; Hillyer, C.D.; Roback, J.D.; Zimring, J.C. Recipient inflammation affects the frequency and magnitude of immunization to transfused red blood cells. Transfusion 2006, 46, 1526-1536.

18. Cognasse, F.; Boussoulade, F.; Chavarin, P.; Acquart, S.; Fabrigli, P.; Lamy, B.; Garraud, O. Release of potential immunomodulatory factors during platelet storage. Transfusion 2006, 46, 1184-1189.

19. Mai, J.; Virtue, A.; Shen, J.; Wang, H.; Yang, X.F. An evolving new paradigm: Endothelial cells -conditional innate immune cells. J. Hematol. Oncol. 2013, 6, 61.

20. Cognasse, F.; Hamzeh-Cognasse, H.; Lafarge, S.; Chavarin, P.; Cogne, M.; Richard, Y.; Garraud, O. Human platelets can activate peripheral blood B cells and increase production of immunoglobulins. Exp. Hematol. 2007, 35, 1376-1387.

21. Hod, E.A.; Spitalnik, S.L. Stored red blood cell transfusions: Iron, inflammation, immunity, and infection. Transfus. Clin. Biol. 2012, 19, 84-89. 
22. Zalpuri, S.; Schonewille, H.; Middelburg, R.; van de Watering, L.; de Vooght, K.; Zimring, J.; van der Bom, J.G.; Zwaginga, J.J. Effect of storage of red blood cells on alloimmunization. Transfusion 2013, 53, 2795-2800.

23. Seftel, M.D.; Growe, G.H.; Petraszko, T.; Benny, W.B.; Le, A.; Lee, C.Y.; Spinelli, J.J.; Sutherland, H.J.; Tsang, P.; Hogge, D.E. Universal prestorage leukoreduction in Canada decreases platelet alloimmunization and refractoriness. Blood 2004, 103, 333-339.

24. van de Watering, L.; Hermans, J.; Witvliet, M.; Versteegh, M.; Brand, A. HLA and RBC immunization after filtered and buffy coat-depleted blood transfusion in cardiac surgery: A randomized controlled trial. Transfusion 2003, 43, 765-771.

25. Slichter, S.J. Leukocyte reduction and ultraviolet B irradiation of platelets to prevent alloimmunization and refractoriness to platelet transfusions. N. Engl. J. Med. 1997, 337, 1861-1869.

26. Marschner, S.; Fast, L.D.; Baldwin, W.M., 3rd; Slichter, S.J.; Goodrich, R.P. White blood cell inactivation after treatment with riboflavin and ultraviolet light. Transfusion 2010, 50, 2489-2498.

27. Bilgin, Y.M.; van de Watering, L.M.; Brand, A. Clinical effects of leucoreduction of blood transfusions. Neth. J. Med. 2011, 69, 441-450.

28. Claas, F.H.; Smeenk, R.J.; Schmidt, R.; van Steenbrugge, G.J.; Eernisse, J.G. Alloimmunization against the MHC antigens after platelet transfusions is due to contaminating leukocytes in the platelet suspension. Exp. Hematol. 1981, 9, 84-89.

29. Semple, J.W.; Speck, E.R.; Cosgrave, D.; Lazarus, A.H.; Blanchette, V.S.; Freedman, J. Extreme leukoreduction of major histocompatibility complex class II positive B cells enhances allogeneic platelet immunity. Blood 1999, 93, 713-720.

30. Gilson, C.R.; Cadwell, C.M.; Smith, N.H.; Hendrickson, J.E.; Zimring, J.C. MHC II on transfused murine blood is not required for alloimmunization against MHC I. Vox Sang. 2010, 99, 369-374.

31. Semple, J.W.; Speck, E.R.; Milev, Y.P.; Blanchette, V.; Freedman, J. Indirect allorecognition of platelets by $\mathrm{T}$ helper cells during platelet transfusions correlates with anti-major histocompatibility complex antibody and cytotoxic T lymphocyte formation. Blood 1995, 86, 805-812.

32. Blajchman, M.A. Transfusion-associated immunomodulation and universal white cell reduction: Are we putting the cart before the horse? Transfusion 1999, 39, 665-670.

33. Aslam, R.; Speck, E.R.; Kim, M.; Freedman, J.; Semple, J.W. Transfusion-related immunomodulation by platelets is dependent on their expression of MHC Class I molecules and is independent of white cells. Transfusion 2008, 48, 1778-1786.

34. Sayeh, E.; Sterling, K.; Speck, E.; Freedman, J.; Semple, J.W. IgG antiplatelet immunity is dependent on an early innate natural killer cell-derived interferon-gamma response that is regulated by CD8+ T cells. Blood 2004, 103, 2705-2709.

35. Vamvakas, E.C.; Blajchman, M.A. Transfusion-related immunomodulation (TRIM): An update. Blood Rev. 2007, 21, 327-348.

36. van Twuyver, E.; Mooijaart, R.J.; ten Berge, I.J.; van der Horst, A.R.; Wilmink, J.M.; Kast, W.M.; Melief, C.J.; de Waal, L.P. Pretransplantation blood transfusion revisited. N. Engl. J. Med. 1991, 325, 1210-1213. 
37. Hendrickson, J.E.; Chadwick, T.E.; Roback, J.D.; Hillyer, C.D.; Zimring, J.C. Inflammation enhances consumption and presentation of transfused RBC antigens by dendritic cells. Blood 2007, 110, 2736-2743.

38. Smith, N.H.; Hod, E.A.; Spitalnik, S.L.; Zimring, J.C.; Hendrickson, J.E. Transfusion in the absence of inflammation induces antigen-specific tolerance to murine RBCs. Blood 2012, 119, $1566-1569$.

39. Zimring, J.C.; Stowell, S.R.; Johnsen, J.M.; Hendrickson, J.E. Effects of genetic, epigenetic, and environmental factors on alloimmunization to transfused antigens: Current paradigms and future considerations. Transfus. Clin. Biol. 2012, 19, 125-131.

40. Papay, P.; Hackner, K.; Vogelsang, H.; Novacek, G.; Primas, C.; Reinisch, W.; Eser, A.; Mikulits, A.; Mayr, W.R.; Kormoczi, G.F. High risk of transfusion-induced alloimmunization of patients with inflammatory bowel disease. Am. J. Med. 2012, 125, 717.e1-717.e8.

41. Tatari-Calderone, Z.; Minniti, C.P.; Kratovil, T.; Stojakovic, M.; Vollmer, A.; Barjaktarevic, I.; Zhang, E.; Hoang, A.; Luban, N.L.; Vukmanovic, S. rs660 polymorphism in Ro52 (SSA1; TRIM21) is a marker for age-dependent tolerance induction and efficiency of alloimmunization in sickle cell disease. Mol. Immunol. 2009, 47, 64-70.

42. Tatari-Calderone, Z.; Tamouza, R.; Le Bouder, G.P.; Dewan, R.; Luban, N.L.; Lasserre, J.; Maury, J.; Lionnet, F.; Krishnamoorthy, R.; Girot, R.; et al. The association of CD81 polymorphisms with alloimmunization in sickle cell disease. Clin. Dev. Immunol. 2013, 2013, 937846.

43. Yazer, M.H.; Triulzi, D.J.; Shaz, B.; Kraus, T.; Zimring, J.C. Does a febrile reaction to platelets predispose recipients to red blood cell alloimmunization? Transfusion 2009, 49, 1070-1075.

44. Aygun, B.; Padmanabhan, S.; Paley, C.; Chandrasekaran, V. Clinical significance of RBC alloantibodies and autoantibodies in sickle cell patients who received transfusions. Transfusion 2002, 42, 37-43.

45. Yazdanbakhsh, K.; Ware, R.E.; Noizat-Pirenne, F. Red blood cell alloimmunization in sickle cell disease: Pathophysiology, risk factors, and transfusion management. Blood 2012, 120, 528-537.

46. Bao, W.; Yu, J.; Heck, S.; Yazdanbakhsh, K. Regulatory T-cell status in red cell alloimmunized responder and nonresponder mice. Blood 2009, 113, 5624-5627.

47. Yu, J.; Heck, S.; Yazdanbakhsh, K. Prevention of red cell alloimmunization by CD25 regulatory T cells in mouse models. Am. J. Hematol. 2007, 82, 691-696.

48. Bao, W.; Zhong, H.; Manwani, D.; Vasovic, L.; Uehlinger, J.; Lee, M.T.; Sheth, S.; Shi, P.; Yazdanbakhsh, K. Regulatory B-cell compartment in transfused alloimmunized and nonalloimmunized patients with sickle cell disease. Am. J. Hematol. 2013, 88, 736-740.

49. Bao, W.; Zhong, H.; Li, X.; Lee, M.T.; Schwartz, J.; Sheth, S.; Yazdanbakhsh, K. Immune regulation in chronically transfused allo-antibody responder and nonresponder patients with sickle cell disease and beta-thalassemia major. Am. J. Hematol. 2011, 86, 1001-1006.

50. Aslam, R.; Hu, Y.; Gebremeskel, S.; Segel, G.B.; Speck, E.R.; Guo, L.; Kim, M.; Ni, H.; Freedman, J.; Semple, J.W. Thymic retention of CD4+CD25+FoxP3+ T regulatory cells is associated with their peripheral deficiency and thrombocytopenia in a murine model of immune thrombocytopenia. Blood 2012, 120, 2127-2132. 
51. Castellino, S.M.; Combs, M.R.; Zimmerman, S.A.; Issitt, P.D.; Ware, R.E. Erythrocyte autoantibodies in paediatric patients with sickle cell disease receiving transfusion therapy: Frequency, characteristics and significance. Br. J. Haematol. 1999, 104, 189-194.

52. Taaning, E.; Tonnesen, F. Pan-reactive platelet antibodies in post-transfusion purpura. Vox Sang. 1999, 76, 120-123.

53. Lopes, L.B.; Fabron-Jr, A.; Chiba, A.K.; Ruiz, M.O.; Bordin, J.O. Impact of using different laboratory assays to detect human leukocyte antigen antibodies in female blood donors. Transfusion 2010, 50, 902-908.

54. Dreyfus, M.; Kaplan, C.; Verdy, E.; Schlegel, N.; Durand-Zaleski, I.; Tchernia, G., Frequency of immune thrombocytopenia in newborns: A prospective study. Blood 1997, 89, 4402-4406.

55. Skogen, B.; Husebekk, A.; Killie, M.K.; Kjeldsen-Kragh, J. Neonatal alloimmune thrombocytopenia is not what it was: A lesson learned from a large prospective screening and intervention program. Scand. J. Immunol. 2009, 70, 531-534.

56. Ahlen, M.T.; Husebekk, A.; Killie, M.K.; Kjeldsen-Kragh, J.; Olsson, M.L.; Skogen, B. The development of severe neonatal alloimmune thrombocytopenia due to anti-HPA-1a antibodies is correlated to maternal ABO genotypes. Clin. Dev. Immunol. 2012, 2012, 156867.

57. Basu, S.; Kaur, R.; Kaur, G. Hemolytic disease of the fetus and newborn: Current trends and perspectives. Asian J. Transfus. Sci. 2011, 5, 3-7.

58. Mawas, F.; Wiener, E.; Williamson, L.M.; Rodeck, C.H. Immunoglobulin G subclasses of antihuman platelet antigen 1a in maternal sera: Relation to the severity of neonatal alloimmune thrombocytopenia. Eur. J. Haematol. 1997, 59, 287-292.

59. Jackman, R.P.; Deng, X.; Bolgiano, D.; Utter, G.H.; Schechterly, C.; Lebedeva, M.; Operskalski, E.; Luban, N.L.; Alter, H.; Busch, M.P.; et al. Leukoreduction and ultraviolet treatment reduce both the magnitude and the duration of the HLA antibody response. Transfusion 2013, doi: $10.1111 / \operatorname{trf} .12317$.

60. Kaneku, H. 2012 annual literature review of donor-specific HLA antibodies after organ transplantation. Clin. Transpl. 2012, 207-217.

61. Loupy, A.; Hill, G.S.; Jordan, S.C. The impact of donor-specific anti-HLA antibodies on late kidney allograft failure. Nat. Rev. Nephrol. 2012, 8, 348-357.

62. Scornik, J.C.; Guerra, G.; Schold, J.D.; Srinivas, T.R.; Dragun, D.; Meier-Kriesche, H.U. Value of posttransplant antibody tests in the evaluation of patients with renal graft dysfunction. Am. J. Transplant. 2007, 7, 1808-1814.

63. Higgins, R.; Hathaway, M.; Lowe, D.; Lam, F.; Kashi, H.; Tan, L.C.; Imray, C.; Fletcher, S.; Zehnder, D.; Chen, K.; et al. Blood levels of donor-specific human leukocyte antigen antibodies after renal transplantation: Resolution of rejection in the presence of circulating donor-specific antibody. Transplantation 2007, 84, 876-884.

64. Platt, J.L. Antibodies in transplantation. Discov. Med. 2010, 10, 125-133.

65. Arnold, M.L.; Heinemann, F.M.; Horn, P.; Ziemann, M.; Lachmann, N.; Muhlbacher, A.; Dick, A.; Ender, A.; Thammanichanond, D.; Fischer, G.F.; et al. 16(th) IHIW: Anti-HLA alloantibodies of the of IgA isotype in re-transplant candidates. Int. J. Immunogenet. 2013, 40, 17-20. 
66. Lowe, D.; Higgins, R.; Zehnder, D.; Briggs, D.C. Significant IgG subclass heterogeneity in HLA-specific antibodies: Implications for pathogenicity, prognosis, and the rejection response. Hum. Immunol. 2013, 74, 666-672.

67. Takiguchi, M.; Nishimura, I.; Hayashi, H.; Karakl, S.; Kariyone, A.; Kano, K. The structure and expression of genes encoding serologically undetected HLA-C locus antigens. J. Immunol. 1989, $143,1372-1378$.

68. Jolly, E.C.; Key, T.; Rasheed, H.; Morgan, H.; Butler, A.; Pritchard, N.; Taylor, C.J.; Clatworthy, M.R. Preformed donor HLA-DP-specific antibodies mediate acute and chronic antibodymediated rejection following renal transplantation. Am. J. Transplant. 2012, 12, 2845-2848.

69. Muro, M.; Sanchez-Bueno, F.; Marin, L.; Torio, A.; Moya-Quiles, M.R.; Minguela, A.; Ramirez, P.; Alemany, J.M.; Miras, M.; Perez-Lopez, M.J.; et al. DQA1 and DQB1 genes polymorphism on acute rejection development in liver transplantation. Transplant. Proc. 2002, 34, 3302-3303.

70. Marrari, M.; Duquesnoy, R.J. Detection of donor-specific HLA antibodies before and after removal of a rejected kidney transplant. Transpl. Immunol. 2010, 22, 105-109.

71. Kraus, E.S.; Parekh, R.S.; Oberai, P.; Lepley, D.; Segev, D.L.; Bagnasco, S.; Collins, V.; Leffell, M.; Lucas, D.; Rabb, H.; et al. Subclinical rejection in stable positive crossmatch kidney transplant patients: Incidence and correlations. Am. J. Transplant. 2009, 9, 1826-1834.

72. Laperrousaz, S.; Tiercy, J.M.; Villard, J.; Ferrari-Lacraz, S. HLA and non-HLA polymorphisms in renal transplantation. Swiss Med. Wkly. 2012, 142, w13668.

73. Opelz, G. Non-HLA transplantation immunity revealed by lymphocytotoxic antibodies. Lancet 2005, 365, 1570-1576.

74. Terasaki, P.I.; Ozawa, M.; Castro, R. Four-year Follow-up of a Prospective Trial of HLA and MICA Antibodies on Kidney Graft Survival. Am. J. Transplant. 2007, 7, 408-415.

75. Sigdel, T.K.; Sarwal, M.M. Moving beyond HLA: A review of nHLA antibodies in organ transplantation. Hum. Immunol. 2013, 74, 1486-1490.

76. Porcheray, F.; DeVito, J.; Yeap, B.Y.; Xue, L.; Dargon, I.; Paine, R.; Girouard, T.C.; Saidman, S.L.; Colvin, R.B.; Wong, W.; et al. Chronic humoral rejection of human kidney allografts associates with broad autoantibody responses. Transplantation 2010, 89, 1239-1246.

77. Reinsmoen, N.L.; Lai, C.H.; Heidecke, H.; Haas, M.; Cao, K.; Ong, G.; Naim, M.; Wang, Q.; Mirocha, J.; Kahwaji, J.; et al. Anti-angiotensin type 1 receptor antibodies associated with antibody mediated rejection in donor HLA antibody negative patients. Transplantation 2010, 90, 1473-1477.

78. Subramanian, V.; Ramachandran, S.; Klein, C.; Wellen, J.R.; Shenoy, S.; Chapman, W.C.; Mohanakumar, T. ABO-incompatible organ transplantation. Int. J. Immunogenet. 2012, 39, 282-290.

79. Nadarajah, L.; Ashman, N.; Thuraisingham, R.; Barber, C.; Allard, S.; Green, L. Literature review of passenger lymphocyte syndrome following renal transplantation and two case reports. Am. J. Transplant. 2013, 13, 1594-1600.

80. Golshayan, D.; Jiang, S.; Tsang, J.; Garin, M.I.; Mottet, C.; Lechler, R.I. In vitro-expanded donor alloantigen-specific $\mathrm{CD} 4+\mathrm{CD} 25+$ regulatory $\mathrm{T}$ cells promote experimental transplantation tolerance. Blood 2007, 109, 827-835.

81. Dons, E.M.; Raimondi, G.; Cooper, D.K.; Thomson, A.W. Induced regulatory T cells: Mechanisms of conversion and suppressive potential. Hum. Immunol. 2012, 73, 328-334. 
82. Ochando, J.C.; Homma, C.; Yang, Y.; Hidalgo, A.; Garin, A.; Tacke, F.; Angeli, V.; Li, Y.; Boros, P.; Ding, Y.; et al. Alloantigen-presenting plasmacytoid dendritic cells mediate tolerance to vascularized grafts. Nat. Immunol. 2006, 7, 652-662.

83. Wood, K.J.; Bushell, A.; Hester, J. Regulatory immune cells in transplantation. Nat. Rev. Immunol. 2012, 12, 417-430.

84. Liu, Z.; Tugulea, S.; Cortesini, R.; Lederman, S.; Suciu-Foca, N. Inhibition of CD40 signaling pathway in antigen presenting cells by T suppressor cells. Hum. Immunol. 1999, 60, 568-574.

85. Cai, J.; Lee, J.; Jankowska-Gan, E.; Derks, R.; Pool, J.; Mutis, T.; Goulmy, E.; Burlingham, W.J. Minor $\mathrm{H}$ antigen HA-1-specific regulator and effector CD8+ T cells, and HA-1 microchimerism, in allograft tolerance. J. Exp. Med. 2004, 199, 1017-1023.

86. Roncarolo, M.G.; Gregori, S.; Lucarelli, B.; Ciceri, F.; Bacchetta, R. Clinical tolerance in allogeneic hematopoietic stem cell transplantation. Immunol. Rev. 2011, 241, 145-163.

87. Zhang, Z.; Yang, L.; Young, K.; DuTemple, B.; Zhang, L. Identification of a previously unknown antigen-specific regulatory $\mathrm{T}$ cell and its mechanism of suppression. Nat. Med. 2000, 6 , $782-789$.

88. Jukes, J.P.; Wood, K.J.; Jones, N.D. Natural killer T cells: A bridge to tolerance or a pathway to rejection? Transplantation 2007, 84, 679-681.

89. Li, Y.; Koshiba, T.; Yoshizawa, A.; Yonekawa, Y.; Masuda, K.; Ito, A.; Ueda, M.; Mori, T.; Kawamoto, H.; Tanaka, Y.; et al. Analyses of peripheral blood mononuclear cells in operational tolerance after pediatric living donor liver transplantation. Am. J. Transplant. 2004, 4, 2118-2125.

90. Martinez-Llordella, M.; Puig-Pey, I.; Orlando, G.; Ramoni, M.; Tisone, G.; Rimola, A.; Lerut, J.; Latinne, D.; Margarit, C.; Bilbao, I.; et al. Multiparameter immune profiling of operational tolerance in liver transplantation. Am. J. Transplant. 2007, 7, 309-319.

91. Yang, J.; Brook, M.O.; Carvalho-Gaspar, M.; Zhang, J.; Ramon, H.E.; Sayegh, M.H.; Wood, K.J.; Turka, L.A.; Jones, N.D. Allograft rejection mediated by memory T cells is resistant to regulation. Proc. Natl. Acad. Sci. USA 2007, 104, 19954-19959.

92. Steger, U.; Kingsley, C.I.; Karim, M.; Bushell, A.R.; Wood, K.J. CD25+CD4+ regulatory T cells develop in mice not only during spontaneous acceptance of liver allografts but also after acute allograft rejection. Transplantation 2006, 82, 1202-1209.

93. Pallier, A.; Hillion, S.; Danger, R.; Giral, M.; Racape, M.; Degauque, N.; Dugast, E.; AshtonChess, J.; Pettre, S.; Lozano, J.J.; et al. Patients with drug-free long-term graft function display increased numbers of peripheral B cells with a memory and inhibitory phenotype. Kidney Int. 2010, 78, 503-513.

94. Sagoo, P.; Perucha, E.; Sawitzki, B.; Tomiuk, S.; Stephens, D.A.; Miqueu, P.; Chapman, S.; Craciun, L.; Sergeant, R.; Brouard, S.; et al. Development of a cross-platform biomarker signature to detect renal transplant tolerance in humans. J. Clin. Invest. 2010, 120, 1848-1861.

95. Newell, K.A.; Allan, A.; Kirk, A.D.; Gisler, T.D.; Bourcier, K.; Suthanthrian, M.; Burlingham, W.J.; Marks, W.H.; Sanz, I.; Lechler, R.I.; et al. Identification of a B cell signature associated with renal transplant tolerance in humans. J. Clin. Invest. 2010, 120, 1836-1847.

96. Coelho, V.; Saitovitch, D.; Kalil, J.; Silva, H.M. Rethinking the multiple roles of B cells in organ transplantation. Curr. Opin. Organ. Transplant. 2013, 18, 13-21. 
97. Thaunat, O. Pathophysiologic significance of B-cell clusters in chronically rejected grafts. Transplantation 2011, 92, 121-126.

98. Lynch, R.J.; Silva, I.A.; Chen, B.J.; Punch, J.D.; Cascalho, M.; Platt, J.L. Cryptic B cell response to renal transplantation. Am. J. Transplant. 2013, 13, 1713-1723.

99. Kraus, A.K.; Cippa, P.E.; Gaspert, A.; Chen, J.; Edenhofer, I.; Wuthrich, R.P.; Lindenmeyer, M.; Segerer, S.; Fehr, T. Absence of donor CD40 protects renal allograft epithelium and preserves renal function. Transpl. Int. 2013, 26, 535-544.

100. Ripoll, E.; Pluvinet, R.; Torras, J.; Olivar, R.; Vidal, A.; Franquesa, M.; Cassis, L.; Cruzado, J.M.; Bestard, O.; Grinyo, J.M.; et al. In vivo therapeutic efficacy of intra-renal CD40 silencing in a model of humoral acute rejection. Gene Ther. 2011, 18, 945-952.

101. Brent, L.; Brown, J.; Medawar, P.B. Skin transplantation immunity in relation to hypersensitivity. Lancet 1958, 2, 561-564.

102. Girlanda, R.; Kleiner, D.E.; Duan, Z.; Ford, E.A.; Wright, E.C.; Mannon, R.B.; Kirk, A.D. Monocyte infiltration and kidney allograft dysfunction during acute rejection. Am. J. Transplant. 2008, 8, 600-607.

103. Tinckam, K.J.; Djurdjev, O.; Magil, A.B. Glomerular monocytes predict worse outcomes after acute renal allograft rejection independent of C4d status. Kidney Int. 2005, 68, 1866-1874.

104. Ulrich, C.; Heine, G.H.; Gerhart, M.K.; Kohler, H.; Girndt, M. Proinflammatory CD14+CD16+ monocytes are associated with subclinical atherosclerosis in renal transplant patients. Am. J. Transplant. 2008, 8, 103-110.

105. Benoit, M.; Desnues, B.; Mege, J.L. Macrophage polarization in bacterial infections. J. Immunol. 2008, 181, 3733-3739.

106. Mosser, D.M.; Edwards, J.P. Exploring the full spectrum of macrophage activation. Nat. Rev. Immunol. 2008, 8, 958-969.

107. Rowshani, A.T.; Vereyken, E.J. The role of macrophage lineage cells in kidney graft rejection and survival. Transplantation 2012, 94, 309-318.

108. Riquelme, P.; Tomiuk, S.; Kammler, A.; Fandrich, F.; Schlitt, H.J.; Geissler, E.K.; Hutchinson, J.A. IFN-gamma-induced iNOS expression in mouse regulatory macrophages prolongs allograft survival in fully immunocompetent recipients. Mol. Ther. 2013, 21, 409-422.

109. Broichhausen, C.; Riquelme, P.; Geissler, E.K.; Hutchinson, J.A. Regulatory macrophages as therapeutic targets and therapeutic agents in solid organ transplantation. Curr. Opin. Organ. Transplant. 2012, 17, 332-342.

110. Benichou, G.; Valujskikh, A.; Heeger, P.S. Contributions of direct and indirect T cell alloreactivity during allograft rejection in mice. J. Immunol. 1999, 162, 352-358.

111. Gould, D.S.; Auchincloss, H., Jr. Direct and indirect recognition: The role of MHC antigens in graft rejection. Immunol. Today 1999, 20, 77-82.

112. Smyth, L.A.; Afzali, B.; Tsang, J.; Lombardi, G.; Lechler, R.I. Intercellular transfer of MHC and immunological molecules: Molecular mechanisms and biological significance. Am. J. Transplant. 2007, 7, 1442-1449.

113. Herrera, O.B.; Golshayan, D.; Tibbott, R.; Salcido Ochoa, F.; James, M.J.; Marelli-Berg, F.M.; Lechler, R.I. A novel pathway of alloantigen presentation by dendritic cells. J. Immunol. 2004, 173, 4828-4837. 
114. Dolan, B.P.; Gibbs, K.D., Jr.; Ostrand-Rosenberg, S. Tumor-specific CD4+ T cells are activated by "cross-dressed" dendritic cells presenting peptide-MHC class II complexes acquired from cell-based cancer vaccines. J. Immunol. 2006, 176, 1447-1455.

115. Ezzelarab, M.; Thomson, A.W. Tolerogenic dendritic cells and their role in transplantation. Semin. Immunol. 2011, 23, 252-263.

116. Brouard, S.; Pallier, A.; Renaudin, K.; Foucher, Y.; Danger, R.; Devys, A.; Cesbron, A.; GuillotGuegen, C.; Ashton-Chess, J.; Le Roux, S.; et al. The natural history of clinical operational tolerance after kidney transplantation through twenty-seven cases. Am. J. Transplant. 2012, 12, 3296-3307.

117. Tryphonopoulos, P.; Ruiz, P.; Weppler, D.; Nishida, S.; Levi, D.M.; Moon, J.; Tekin, A.; Velez, M.; Neuman, D.R.; Island, E.; et al. Long-term follow-up of 23 operational tolerant liver transplant recipients. Transplantation 2010, 90, 1556-1561.

118. Roussey-Kesler, G.; Giral, M.; Moreau, A.; Subra, J.F.; Legendre, C.; Noel, C.; Pillebout, E.; Brouard, S.; Soulillou, J.P. Clinical operational tolerance after kidney transplantation. Am. J. Transplant. 2006, 6, 736-746.

119. Heidt, S.; Wood, K.J. Biomarkers of operational tolerance in solid organ transplantation. Expert Opin. Med. Diagn. 2012, 6, 281-293.

120. Thakkinstian, A.; Dmitrienko, S.; Gerbase-Delima, M.; McDaniel, D.O.; Inigo, P.; Chow, K.M.; McEvoy, M.; Ingsathit, A.; Trevillian, P.; Barber, W.H.; et al. Association between cytokine gene polymorphisms and outcomes in renal transplantation: A meta-analysis of individual patient data. Nephrol. Dial. Transplant. 2008, 23, 3017-3023.

121. Burlingham, W.J.; Benichou, G. Bidirectional alloreactivity: A proposed microchimerism-based solution to the NIMA paradox. Chimerism 2012, 3, 29-36.

122. Tsurufuji, M.; Ishiguro, K.; Shinomiya, T.; Uchida, T.; Kamada, N. Immunosuppressive activity of serum from liver-grafted rats: In vitro specific inhibition of mixed lymphocyte reactivity by antibodies against class II RT1 alloantigens. Immunology 1987, 61, 421-428.

123. Nakano, T.; Goto, S.; Lai, C.Y.; Hsu, L.W.; Kao, Y.H.; Lin, Y.C.; Kawamoto, S.; Chiang, K.C.; Ohmori, N.; Goto, T.; et al. Experimental and clinical significance of antinuclear antibodies in liver transplantation. Transplantation 2007, 83, 1122-1125.

124. Nakano, T.; Kawamoto, S.; Lai, C.-Y.; Sasaki, T.; Aki, T.; Shigeta, S.; Goto, T.; Sato, S.; Goto, S.; Chen, C.-L.; et al. Liver transplantation-induced antihistone $\mathrm{H} 1$ autoantibodies suppress mixed lymphocyte reaction. Transplantation 2004, 77, 1595-1603.

125. Nakano, T.; Kawamoto, S.; Lai, C.Y.; Hsu, L.W.; Lin, Y.C.; Sasaki, T.; Aki, T.; Shigeta, S.; Goto, T.; Ohmori, N.; et al. Characterization of immunosuppressive factors expressed in serum by rat tolerogenic liver transplantation. Transplant. Proc. 2005, 37, 80-81.

126. Collin, M.; Ehlers, M. The carbohydrate switch between pathogenic and immunosuppressive antigen-specific antibodies. Exp. Dermatol. 2013, 22, 511-514.

127. Schwab, I.; Nimmerjahn, F. Intravenous immunoglobulin therapy: How does IgG modulate the immune system? Nat. Rev. Immunol. 2013, 13, 176-189.

128. Semple, J.W. gamma -Globulins prepared from sera of multiparous women bind anti-HLA antibodies and inhibit an established in vivo human alloimmune response. Blood 2002, 100, $1055-1059$. 
129. Oefner, C.M.; Winkler, A.; Hess, C.; Lorenz, A.K.; Holecska, V.; Huxdorf, M.; Schommartz, T.; Petzold, D.; Bitterling, J.; Schoen, A.-L.; et al. Tolerance induction with T cell-dependent protein antigens induces regulatory sialylated IgGs. J. Allergy Clin. Immunol. 2012, 129, 1647-1655.

130. Brouard, S.; Mansfield, E.; Braud, C.; Li, L.; Giral, M.; Hsieh, S.C.; Baeten, D.; Zhang, M.; Ashton-Chess, J.; Braudeau, C.; et al. Identification of a peripheral blood transcriptional biomarker panel associated with operational renal allograft tolerance. Proc. Natl. Acad. Sci. USA 2007, 104, 15448-15453.

(C) 2014 by the authors; licensee MDPI, Basel, Switzerland. This article is an open access article distributed under the terms and conditions of the Creative Commons Attribution license (http://creativecommons.org/licenses/by/3.0/). 